\title{
Frieden trotz Umbruch: Eine Analyse der ausbleibenden Gewalteskalation in Boliviens »demokratischer Revolution«
}

Seit 2006 durchläuft Bolivien einen tiefgreifenden politischen Umbruch. Dieser war und ist überaus konfliktträchtig - die Auseinandersetzungen um eine neue Verfassung führten das Land 2008 an den Rand eines Bürgerkriegs. Gleichwohl blieb das Ausmaß politischer Gewalt gering, so dass der Veränderungsprozess bisher weitgehend friedlich und in einem grundlegend demokratischen Rahmen verlief. Dies ist aus friedens- und konflikttheoretischer Sicht erklärungsbedürftig, bedenkt man das Ausmaß an Umverteilung politischer Macht in einem Kontext, in dem in einer Situation ohnehin schwacher demokratischer Institutionen die etablierten politischen Spielregeln offen in Frage gestellt wurden. Der Beitrag fragt nach den Ursachen, die den innerbolivianischen Frieden trotz Umbruch erklären. Die Analyse verweist auf die Bedeutung der Friedensleistungen der Demokratie, zeigtaber, dass demokratische Institutionen in ihrer Wirkung ambivalent sind und ihre Fähigkeit zur friedlichen Bearbeitung soziopolitischer Konflikte nur zu verstehen ist, wenn sie als eingebettet in und im Zusammenspiel mit informellen Politikmustern, politökonomischen Konfliktkonstellationen und internationalen Kontextbedingungen betrachtet werden.

Schlagworte: Friedensursachen, Demokratisierung, democratic civil peace, Konfliktbearbeitung, Lateinamerika

\section{Einleitung}

Zeiten tiefgreifender Umbrüche sind Zeiten erhöhten Gewaltrisikos. ${ }^{1}$ Dies gilt, so die einschlägige Forschung zu den Ursachen politischer Gewalt, namentlich für

1 Eine frühere Version dieses Beitrags wurde auf dem Jahreskolloquium der AFK »Widerstand Gewalt - Umbruch: Bedingungen gesellschaftlichen Wandels«, 22.-24. März 2012, Evangelische Akademie Villigst, präsentiert. Ich danke den TeilnehmerInnen sowie zwei anonymen Gutachtern für Fragen und Anregungen. 
Umbrüche, die die politische Ordnung eines Landes betreffen. ${ }^{2}$ Allerdings ist nicht erst seit der so genannten »dritten Welle« der Demokratisierung ebenso klar, dass politische Transformationsprozesse auch weitgehend friedlich verlaufen können (Huntington 1991). Damit stellt sich die Frage, unter welchen Bedingungen politische Umbrüche einen friedlichen, wann einen gewaltsamen Verlauf haben.

Die Forschung hat sich dieser Frage zumeist mit spezifischem Blick auf Episoden von Regimewechseln (Demokratisierung, Autokratisierung) angenommen. Dabei setzt sie erstens primär auf quantitative large $N$-Studien und fokussiert zweitens auf die Abwesenheit bzw. den Bruch des Friedens, sprich: auf Fälle, in denen Umbrüche zu massiver politischer Gewalt führen (Wolff 2009: 999; Spanger/Schesterinia 2012). Beides - die Dominanz quantitativ operierender Studien und der Fokus auf Gewalt/Krieg - charakterisiert allgemein die Forschung zu den Ursachen von Gewalt und Frieden im innergesellschaftlichen Raum (vgl. Ballentine/Sherman 2003; Bussmann et al. 2009; Jung 2005). Die Klage von Volker Matthies (1994), dass die »Friedensursachenforschung « zur Frage, warum und wie Kriege enden, ein »vernachlässigtes Forschungsfeld « darstelle, ist so zwar mittlerweile verjährt. Allerdings konzentriert sich die Untersuchung von Friedensursachen genau im Sinne Matthies' auf die Frage der Beendigung von Gewalt und, mit Blick auf die Thematik politischer Umbrüche, insbesondere auf Demokratisierung nach Bürgerkriegen bzw. Chancen und Grenzen des liberal peacebuilding (vgl. Gromes 2007; Newman et al. 2009). Eine Friedensursachenforschung, die sich Fällen annimmt, in denen es gar nicht erst zur Eskalation politischer Gewalt kam, findet dagegen nach wie vor kaum statt. Darum soll es in diesem Artikel gehen, der den tiefgreifenden, aber bisher dennoch weitgehend friedlichen politischen Umbruch untersucht, den Bolivien seit 2006 durchläuft. Damit ist zugleich ein qualitativer, hier gar auf den Einzelfall bezogener Zugang gewählt, der im Unterschied zum statistischen Fokus auf Korrelationen und »kausale« Effekte darauf zielt, kausale Mechanismen herauszuarbeiten.

Im Folgenden werde ich zunächst einen knappen Überblick über die politischen Veränderungen geben, die sich in Bolivien seit der Jahrtausendwende entfaltet haben (2.), und darauf aufbauend begründen, warum Bolivien im Sinne der Fragestellung als signifikantes Nicht-Ereignis von Interesse ist: als Nicht-Ereignis, insofern der von Präsident Evo Morales seit 2006 vorangetriebene Umbruch bisher

2 Als Gewalt bzw. Bürgerkrieg verursachende Faktoren, die einen Umbruch der politischen Ordnung betreffen, betont die Forschung dabei einerseits Prozesse des Regimewandels wie Demokratisierung und Autokratisierung, andererseits politische Instabilität im Allgemeinen (vgl. Bussmann et al. 2009: 21; Cederman et al. 2010; Fearon/Laitin 2003; Hegre et al. 2001; Mansfield/Snyder 2008; Sambanis 2005: 315-316). 
weitgehend friedlich verlief; als signifikant, weil der Stand der Forschung zu den Ursachen politischer Gewalt einen gewaltsamen Verlauf durchaus nahegelegt hätte (3.).

Um den gewaltarmen Verlauf des Umbruchs zu erklären, verfolgt der vorliegende Beitrag eine analytisch-eklektizistische Strategie. In der Formulierung von Rudra Sil und Peter Katzenstein (2010: 20) zielt analytischer Eklektizismus darauf, erklärungsbedürftige politische Phänomene in ihrer Komplexität zu reflektieren und dafür »die Vielfalt, Heterogenität und Interaktion kausaler Mechanismen und Prozesse « in den Blick zu nehmen. Anstatt konkurrierende Theorien und Paradigmen gegeneinander zu testen, geht es mithin darum, komplexe, problemfokussierte Erklärungen für ein gegebenes empirisches Phänomen zu entwickeln. ${ }^{3}$ Konkret bietet es sich an, mit dem vielversprechendsten Erklärungsansatz zu beginnen und dessen Reichweite und Grenzen empirisch auszuloten, um ihn dann sukzessive - soweit für die Erklärung nötig - um weitere Elemente zu ergänzen. In diesem Sinne beginnt Abschnitt 4 mit dem Ansatz des democratic civil peace, der eine klare und intuitiv plausible Erklärung anbietet: Demnach sollte der demokratische Charakter des politischen Systems in Bolivien die Fortdauer des innergesellschaftlichen Friedens verursachen. In der Tat erweisen sich die zwei Kausalmechanismen demokratischer Konfliktentschärfung (4.1) und demokratischer Konfliktlinderung (4.2) als bedeutend, die Analyse zeigt aber zugleich deutliche Grenzen einer solchen demokratiezentrierten Erklärung (4.3). Deshalb werden drei weitere Kausalmechanismen herangezogen: informelle Formen der Konfliktbearbeitung (4.4), die politökonomische Opportunitätsstruktur (4.5) sowie der internationale Kontext (4.6).

Die theoretischen Ansätze und Forschungsergebnisse der Friedens- und Konfliktforschung, auf die der vorliegende Beitrag zurückgreift, werden jeweils dort eingeführt, wo sie für die Entwicklung der Argumentation benötigt werden: die Forschung zu Gewalt- und Bürgerkriegsursachen in Abschnitt 3, um den Umbruch in Bolivien als potenziell gewaltträchtig auszuweisen; die Forschung zu Friedensursachen in Abschnitt 4, um die verschiedenen Kausalmechanismen zu begründen. Dabei beziehe ich mich insbesondere in Abschnitt 4 auf theoretische Überlegungen, die ich an anderer Stelle ausführlicher entfaltet habe (vgl. Wolff 2008; 2009). Empirisch fokussiert der Beitrag auf den Großkonflikt zwischen dem Regierungslager um Präsident Evo Morales und der Opposition um die »alten Eliten« und die regionalen Autonomiebewegungen, um die Komplexität multipler Konflikte in Bolivien

3 Ziel analytisch-eklektizistischer Forschung ist nicht die Etablierung schlanker Theorien mit umfassendem Erklärungsanspruch, sondern die Entwicklung einer komplexen kausalen Argumentation, die auf der Ebene von Theorien mittlerer Reichweite angesiedelt ist (Sil/Katzenstein 2010: 208). 
für die Analyse handhabbar zu machen. Im Zentrum steht der konfliktträchtige Aushandlungsprozess zwischen diesen beiden Konfliktparteien, der sich auf die von Morales initiierte verfassungsrechtliche »Neugründung « Boliviens bezog. Untersuchungszeitraum ist damit die erste Amtszeit von Präsident Morales (2006-2009), die mit der Annahme einer neuen Verfassung und Neuwahlen endete (siehe 2.). Lediglich im Schlusskapitel (5.) wird ein kurzer Blick auf jüngere Entwicklungen geworfen.

\section{Die »demokratische Revolution« des Evo Morales}

Nach einer turbulenten Transition zur Demokratie wurde Bolivien nach $1985 \mathrm{zu}$ einem viel gelobten Modell, das Demokratisierung, Stabilisierung und neoliberale Strukturanpassung erfolgreich zu verbinden schien (vgl. Mayorga 1997; Puhle 2001). Zwischen 2000 und 2005 kam es jedoch zu einer wellenartigen Eskalation sozialer Konflikte, die 2003 im protestförmigen Sturz von Präsident Sánchez de Lozada gipfelte. Im Zuge der Massenproteste etablierte sich der Gewerkschaftsführer, Kokabauer und Kopf der Bewegung zum Sozialismus (Movimiento al Socialismo, MAS) Evo Morales als Leitfigur der sozialen und indigenen Bewegungen des Landes. Im Dezember 2005 wurde Morales mit absoluter Mehrheit zum ersten indigenen Präsidenten Boliviens gewählt. Seit Amtsantritt hat die MAS-Regierung weitreichende politische Veränderungen eingeleitet, insbesondere eine grundlegende Verfassungsreform sowie Richtungswechsel in der Wirtschafts- und Sozialpolitik, der Drogenbekämpfung und der Außenpolitik. ${ }^{4}$

Die »Neugründung« Boliviens über eine Verfassunggebende Versammlung (Asamblea Constituyente) entsprach einer historischen Forderung der indigenen Bewegungen des Landes. Die Versammlung wurde im Juli 2006 gewählt und schloss ihre Arbeit im Dezember 2007 ab. Die Annahme des Verfassungsentwurfs mit Zweidrittelmehrheit der anwesenden Mitglieder - bei Abwesenheit der wichtigsten Oppositionsparteien - war jedoch höchst umstritten. Insbesondere in den Departments des südöstlichen Tieflandes, in dem die Opposition gegen Morales ihre Hochburg hat, mobilisierten regionale Autonomiebewegungen gegen die Regierung. Erst nach Monaten teils heftiger politischer Auseinandersetzung gelang im Oktober 2008 ein Abkommen im Kongress, der mit Zweidrittelmehrheit eine de-

4 Einen Überblick über Bolivien unter Evo Morales bieten: Crabtree/Whitehead (2008); Ernst/ Schmalz (2009); Gamarra (2008); Kohl/Bresnahan (2010); Lessmann (2010); PNUD (2010 a; 2010 b); Zegada et al. (2011). 
taillierte Überarbeitung der Verfassungsvorlage vornahm (vgl. Romero et al. 2009)..$^{5}$ Die neue Magna Charta erklärt Bolivien zu einem »plurinationalen Staat«, beinhaltet entsprechend weitreichende indigene (Kollektiv-)Rechte und bedeutet allgemein einen tiefgreifenden normativen und institutionellen Wandel, der die repräsentative Demokratie um direktdemokratische, partizipative und $\gg$ kommunitaristische « Elemente ergänzt, den politisch-bürgerlichen Rechten weitgehende wirtschaftliche, soziale und kulturelle Menschenrechte zur Seite stellt und die private Marktwirtschaft in eine gemischte Wirtschaftsstruktur einordnet (vgl. Wolff 2012). Im Januar 2009 erhielt die neue Verfassung in einem Referendum über $60 \% \mathrm{Zu}$ stimmung. Ende 2009 folgten Neuwahlen, bei denen Morales klar im Amt bestätigt wurde und der MAS eine Zweidrittelmehrheit im neuen Parlament, der Asamblea Legislativa Plurinacional, gewann. Seit 2010 wurde eine Reihe von Organgesetzen zur Implementierung der neuen Verfassung verabschiedet. ${ }^{6}$

Im Bereich der Wirtschafts- und Sozialpolitik hatte Morales einen Bruch mit neoliberalen Rezepten versprochen. Entsprechend baute die Regierung die ökonomische Rolle des Staates aus. Im Zuge der im Mai 2006 verkündeten »Nationalisierung des Erdgases« wurden mit den betroffenen internationalen Unternehmen neue Verträge ausgehandelt, die staatliche Kontrolle des Sektors ausgeweitet, das staatliche Erdgasunternehmen Yacimientos Petroliferos Fiscales Bolivianos (YPFB) wiederbelebt und Steuern auf die Erdgasförderung erhöht. Steigende Staatseinnahmen, insbesondere aus Erdgas- und Minenausbeutung, flossen in ausgeweitete Sozialausgaben und öffentliche Investitionen (Weisbrot et al. 2009; vgl. Kaup 2010; Mendonça/Santaella 2010). Darüber hinaus kündigte die Regierung Morales eine "zweite Bodenreform « an, intensivierte die Umverteilung von Land und verschärfte die entsprechenden gesetzlichen und verfassungsrechtlichen Vorgaben (vgl. Ströbele-Gregor 2009; Urioste 2009). Schließlich wendete sich Morales gegen die bisher von den USA forcierte erzwungene Kokavernichtung und legalisierte den Anbau von Koka (begleitet von einer Strategie der kooperativen Anbaubegrenzung), setzte aber staatliche Maßnahmen gegen Drogenproduktion und -handel fort (vgl. Farthing/Kohl 2010).

Die erste Amtszeit von Präsident Morales (2006-2009) steht damit für einen bemerkenswerten Prozess politischen Wandels, der vielleicht keine »Revolution« im

5 Während die Verfassunggebende Versammlung speziell zur Ausarbeitung einer neuen Verfassung gewählt worden war, besaß der Kongress - das reguläre nationale Parlament im Rahmen der alten Verfassung - keinerlei formale Autorität zur Überarbeitung der Verfassungsvorlage. Unter der neuen Verfassung heißt das bolivianische Parlament »Plurinationale Legislative Versammlung«.

$6 \mathrm{Zu}$ Verfassungsprozess und neuer Verfassung vgl. im Überblick Gamarra (2008: 138-141); IDEA Internacional (2010); Käss/Velásquez (2009); Lehoucq (2008); Romero et al. (2009); Wolff (2012). 
eigentlichen Sinne darstellt, aber jedenfalls einen tiefgreifenden politischen Umbruch. Die Regierung des MAS und die neue Verfassung stehen nicht nur symbolisch für die politische Inkorporation der indigenen Bevölkerung des Landes, die zwar rund 60\% der Gesamtbevölkerung Boliviens stellt und bereits in der Revolution von 1952 das Wahlrecht erhielt, aber auch unter demokratischen Vorzeichen bis dato faktisch weitgehend marginalisiert gewesen war. Zwar ist die Selbstbezeichnung der MAS-Regierung als »Regierung der sozialen Bewegungen« sicherlich übertrieben (vgl. Anria 2010; Stefanoni 2009), und seit 2010 sind wachsende Konflikte zwischen Regierung und sozialen und indigenen Bewegungen zu verzeichnen (vgl. Mokrani 2011; Zegada et al. 2011: 93-98). Generell hat sich unter Morales aber doch zweifellos ein weitgehender Wechsel der politischen Eliten vollzogen, indem das bisherige politische Establishment weitgehend entmachtet wurde, die bisher dominanten gesellschaftlichen Kräfte deutlich an Macht eingebüßt haben und die Repräsentation und Partizipation bisher politisch und sozioökonomisch marginalisierter Kräfte massiv zugenommen hat (vgl. PNUD 2010 a; 2010 b; Zegada et al. 2011: 245-246).

\section{Boliviens friedlicher Umbruch als signifikantes Nicht-Ereignis}

Die Literatur zum Verhältnis von Demokratie und politischer Gewalt betont die Gefahren, die von der Ablösung eines autoritären Regimes ausgehen (vgl. Cederman et al. 2010; Hegre et al. 2001; Mansfield/Snyder 2008). Der skizzierte Umbruch in Bolivien stellt allerdings keinen Regimewechsel im Sinne der üblichen Schemata dar. Nach Einschätzung der meisten Experten ist das politische System Boliviens vor, während wie nach der Verfassungsänderung grundsätzlich demokratisch strukturiert; der oben umrissene Umbruch verlief mithin im Rahmen einer bestehenden Demokratie (vgl. z.B. Bertelsmann Stiftung 2009; Gamarra 2008; Wolff 2012). Dieses Bild bestätigen auch die gängigen Demokratie-Indices, die für Bolivien nach 2005 keine signifikanten Tendenzen der Demokratisierung (oder Autokratisierung) verzeichnen. ${ }^{7}$ Damit ist jedoch das Ausbleiben einer gewaltförmigen Konflikteskalation in Bolivien keineswegs trivial, betont doch etwa Nicholas Sambanis »das

7 Der Bertelsmann Transformation Index (BTI) stuft Bolivien bei graduellen Schwankungen und Verschiebungen als »defekte« (illiberale und delegative) Demokratie ein (Bertelsmann Stiftung 2009: 2). Bei Freedom House gilt das Land als partiell freie elektorale Demokratie (Freedom House 2011). Nach Polity IV, das politische Regime auf einer Skala von -10 (umfassend autokratisch) bis +10 (umfassend demokratisch) verortet, bleibt Bolivien trotz einer Abstufung von +8 auf +7 im demokratischen Spektrum ( +6 bis +10 ) (Marshall/Jaggers 2011). 
erhöhte Risiko politischer Gewalt, das von einem Machtübergang auch ohne Regimeübergang ausgehen kann« (Sambanis 2005: 315; Hervorh. im Orig.) ${ }^{8}$

Ein solcher Machtübergang lässt sich für Bolivien eindeutig konstatieren. Die Wahl von Morales und die sich anschließende Regierung des MAS bedeuteten eine dramatische Umverteilung politischer Macht und bedrohten entsprechend unmittelbar vitale Interessen der bisherigen Eliten (vgl. Eaton 2011: 300-302). So wurden die Parteien der politischen Elite, die seit 1985 die demokratischen Institutionen des Landes unumstritten dominiert hatten, mit den Parlamentswahlen 2005 und den Wahlen zur Verfassunggebenden Versammlung 2006 zu einer oppositionellen Minderheit, die sich einer Mehrheit der »anti-systemischen « Partei MAS gegenüber sah. Mit dieser Kräfteverschiebung innerhalb der politischen Institutionen ging zugleich ein drastischer Machtverlust der mit den »traditionellen « Parteien eng verwobenen sozioökonomischen Eliten des Landes einher. So verloren die Unternehmerverbände ihren gewohnt privilegierten Zugang zur Politik und sahen sich stattdessen mit der anti-neoliberalen, auf »Nationalisierung « und Stärkung des Staates setzenden Wirtschaftspolitik des MAS konfrontiert. Gleiches gilt für das Agrobusiness und namentlich die Großgrundbesitzer im südöstlichen Tiefland, die Enteignung oder jedenfalls Landumverteilung fürchten mussten. ${ }^{9}$ Hinzu kam die generelle Befürchtung, die auch Teile der »weißen«, städtischen Mittelschichten insbesondere im südöstlichen Tiefland teilten, dass mit dem Wahlsieg von Morales und MAS nun die arme, indigene Bevölkerung des Hochlandes die Macht im Staat übernommen habe, um diesen in einem umgedrehten Rassismus nun nach ihrer Maßgabe umzugestalten. ${ }^{10}$

Gleichzeitig bestand aus Sicht der Opposition gegen die neue MAS-Regierung wenig Anlass, sich auf den Schutz und die Vermittlungsfunktion staatlicher Institutionen und demokratischer Verfahren zu verlassen. Denn erstens sah sie sich seit

8 Als Beispiel nennt er einen Führungswechsel innerhalb eines fortgesetzt autokratischen Regimes, aber die potenziellen Implikationen eines Elitenwechsels können grundsätzlich ebenso ein demokratisches Regime betreffen: »Disaffected elites with access to war-making capital may strike at the new leadership and a civil war can occur from a military coup, particularly if the military splits, each supporting a faction of elites « (Sambanis 2005: 315). Dass Eliten, die sich von einer neuen (gewählten) Regierung bedroht sehen, als Gegenwehr zu undemokratischen und gewaltträchtigen Mitteln greifen, ist aus der Geschichte des Zusammenbruchs lateinamerikanischer Demokratien nur zu gut bekannt (vgl. Linz 1978; Rueschemeyer et al. 1992: Kap. 5).

9 Nach der Revolution 1952 kam es zu einer Agrarreform, die Großgrundbesitz abschaffte. Diese betraf allerdings nicht das Tiefland, das damals noch kaum erschlossen war. Großgrundbesitz und großflächiges Agrobusiness bildeten sich hier erst deutlich später - und existieren entsprechend bis heute (Ströbele-Gregor 2009: 145).

10 Vgl. Isidoro (2009); Peñaranda (2009); Schilling-Vacaflor (2010: 174-212); Urioste (2009); Zegada (2010). 
den Wahlen 2005 systematisch in der Minderheit, unterlag mithin zuverlässig in allen nationalen Wahlen und Referenden. Zweitens signalisierte der MAS und namentlich Präsident Morales nur begrenzten Respekt vor prozeduralen und auch verfassungsmäßigen Beschränkungen, während der gesamte Prozess des politischen Wandels explizit auf den Abbau der bestehenden demokratischen Institutionen zu Gunsten neuer zielte. Drittens war just im Kontext des Umbruchsprozesses die Judikative als Kontrollorgan nur eingeschränkt funktionsfähig (vgl. Wolff 2011: 5-6). ${ }^{11}$ Eine zentrale Bedingung, die das Überleben der Demokratie unter Bedingungen kapitalistischer Entwicklung ermöglicht, war damit nicht mehr gegeben: dass die demokratischen Institutionen »die dominanten Klassen gegen wahrgenommene Bedrohungen ihrer vitalen Interessen beschützen« (Rueschemeyer et al. 1992: 78).

Der Umbruch in Bolivien ist damit nicht »nur « als Machtübergang im Sinne von Sambanis (2005: 315) zu charakterisieren, sondern weist zusätzlich eine Reihe weiterer Charakteristika auf, die der Forschung zufolge Regimewandel konfliktträchtig machen und die Friedensfähigkeit der Demokratie unterminieren: ${ }^{12}$ Die politischen Spielregeln wurden grundlegend hinterfragt. Generell sind die staatlichen Institutionen Boliviens schwach, und den gestiegenen Partizipationsansprüchen stand kein institutionalisiertes Parteiensystem gegenüber. Hinzu kommen ein niedriges Niveau ökonomischer Entwicklung, hohe Armuts- und Ungleichheitsraten sowie diverse hier: ethnische, soziale, politische und regionale - cleavages, die sich tendenziell überlappen. Unter solchen Bedingungen, so prominent Mansfield und Snyder (2008: 4), dienen demokratische Wahlen häufig nicht der Befriedung des Konfliktaustrags, sondern werden zum Instrument der politischen Auseinandersetzung (vgl. Snyder 2000; Wimmer/Schetter 2003: 254). Paul Collier (2009: 239) zählt Bolivien explizit zu den Ländern der »Bottom Billion«, in denen nach seinen statistischen Berechnungen die Demokratie - und konkret Wahlen - ein überaus riskantes Geschäft sind. Generell gerät die Fähigkeit der Demokratie zur Aufrechterhaltung des innergesellschaftlichen Friedens an ihre Grenzen, wenn konfliktträchtige cleavages nicht mehr hinreichend durch einen basalen Konsens über »universal akzeptierte Grenzen« (Diamond 1990: 49) des Konfliktaustrags gemäßigt werden.

11 Konflikte zwischen der Regierung Morales und den obersten Gerichten führten dazu, dass die Judikative in einer Reihe von Rücktritten nach und nach ihre Handlungs- und Entscheidungsfähigkeit verlor (vgl. Bertelsmann Stiftung 2009). Erst 2010 erfolgte eine temporäre Neubesetzung der obersten Gerichte, 2011 dann die Wahl neuer Richter (gemäß neuer Verfassung per Direktwahl).

12 Vgl. Collier (2009); Diamond (1990); Gromes (2007: 70-93); Huntington (1970); Mansfield/Snyder (2008); Sambanis (2005); Snyder (2000); Wimmer/Schetter (2003). 
Eine gewaltsame Eskalation des Konflikts um das von Morales vorangetriebene Transformationsprojekt hätte im Sinne der Forschung über politische Gewalt und Bürgerkriegsursachen also durchaus nahe gelegen. Dass dies nicht bloß Theorie ist, zeigte sich im September 2008, als die Konfrontation zwischen nationaler Regierung und regional konzentrierter Opposition in einer offenen politischen Krise gipfelte und das Land an den Rand eines Bürgerkriegs brachte (Böhrt 2009: 54; Jost 2009: 38). Die regionalen Autonomiebewegungen in den südöstlichen TieflandDepartments ${ }^{13}$ Boliviens, der so genannten media luna- (Halbmond-) Region, waren nach der Wahl Morales' schnell zum Kern der Opposition geworden. Im Tiefland Boliviens sind die Gasfelder und die Agrarexportwirtschaft konzentriert, womit sowohl die Umverteilung der Erdgaseinnahmen als auch die angestrebte Landreform primär die Departments der media luna betrafen. Generell ist die Region relativ wohlhabend, während der Anteil der indigenen Bevölkerung relativ gering ist. Die Autonomiebewegungen wurden von den gewählten Präfekten der jeweiligen departamentos und regionalen »Bürgerschaftskomitees « (comités cívicos) getragen, die gemeinsam insbesondere die Elite - und konkret die Agrarunternehmer und Großgrundbesitzer - repräsentierten.

Im Mai und Juni 2008 führten die Autonomiebewegungen in den Tiefland-Departments illegale Volksabstimmungen durch, in denen so weitreichende wie verfassungswidrige Autonomiestatute angenommen wurden (Schilling-Vacaflor 2010: 190). Im August rief das Oppositionsbündnis der Tiefland-Departments zu einem Streik gegen die Regierung auf, der sich insbesondere gegen die Umverteilung der Erdgaseinnahmen richtete (Peñaranda 2009: 152-153). Als dies scheiterte, eskalierten die Autonomiebewegungen die Lage: Im September 2008 blockierten oppositionelle Gruppen im Tiefland Straßen und Gas-Pipelines, besetzten und plünderten lokale Institutionen des Zentralstaats. Zwischen oppositionellen und regierungsnahen Gruppierungen kam es zu gewaltsamen Zusammenstößen. Trauriger Höhe- und Wendepunkt der Krise war ein Zusammenstoß zwischen bewaffneten Truppen mit Verbindungen zur oppositionellen Präfektur und regierungsnahen Bauern, der zwischen zehn und 20 Toten (primär auf Seiten der regierungsnahen campesinos) forderte und in einem Untersuchungsbericht der Union Südamerikanischer Nationen (UNASUR) als »Massaker« bezeichnet wurde. In Folge ließ die Regierung Morales den oppositionellen Präfekt von Pando festnehmen und rief den

13 Die Departments (departamentos) bilden die obersten subnationalen Verwaltungseinheiten Boliviens, grob mit den deutschen Bundesländern vergleichbar. An ihrer Spitze steht ein - seit 2005 direkt gewählter - Präfekt bzw. Gouverneur. Die so genannte media luna bilden die vier TieflandDepartments Beni, Pando, Santa Cruz und Tarija. 
Ausnahmezustand über das Department aus. ${ }^{14}$ Im Oktober 2008 gelang der genannte Kompromiss im Kongress, der dem Verfassungsreferendum den Weg ebnete. Die Konflikte zwischen Regierung und Opposition waren damit alles andere als aus der Welt, aber die kurze Krise vom September 2008 blieb doch die (bisher) einzige Phase ernster Gewalteskalation.

In der Summe bleibt das Bild einer weitgehend friedlichen »demokratischen Revolution $«,{ }^{15}$ die nicht zuletzt mit Blick auf die Geschichte gewaltsamer Umstürze in Bolivien bemerkenswert ist. Einschlägige Konfliktindices bestätigen dies. ${ }^{16}$

\section{Versuch einer Erklärung}

Um den friedlichen Verlauf des bolivianischen Umbruchs zu erklären, legt es die eingangs umrissene analytisch-eklektizistische Vorgehensweise nahe, zunächst auf das Paradigma des democratic civil peace zurückzugreifen (Hegre et al. 2001). ${ }^{17}$ Das Ausbleiben breiterer politischer Gewalt wäre demnach schlicht Folge des demokratischen Charakters des politischen Systems. Als Kausalmechanismen, über die die Demokratie den Frieden reproduziert, lassen sich dabei die Entschärfung und die Linderung soziopolitischer Konflikte identifizieren. Konfliktentschärfend wirkt die Demokratie, indem sie Institutionen zur friedlichen Artikulation von gesellschaftlichen Forderungen bzw. zum gewaltlosen Austrag gesellschaftlicher Konflikte bietet, konfliktlindernd, indem diese Institutionen zugleich dafür sorgen, dass drängende politische Probleme politisch aufgenommen bzw. gesellschaftlich artikulierte Konfliktursachen abgebaut werden. In den Begrifflichkeiten der allgemeinen Forschung über politische Gewalt bezieht sich Ersteres auf die politischen Opportunitätsstrukturen, die die Demokratie bietet und die gewaltsamen Wider-

14 Zur Eskalation im September 2008 vgl. Humphreys/Bebbington (2010: 152-153); Peñaranda (2009: 157-161); Romero (2009: 16-17); Schilling-Vacaflor (2010: 196-197). Im Zuge der Proteste der Autonomiebewegungen eskalierte auch die rassistische Gewalt, die sich insbesondere in Santa Cruz gegen die indigene Bevölkerung und ihre Organisationen richtete (vgl. Schilling-Vacaflor 2010: 195-199).

15 Die Qualifikation als »friedlich«, das sei hier der Vollständigkeit halber angemerkt, bezieht sich selbstredend lediglich auf einen negativen oder engen Friedensbegriff.

16 Das UCDP/PRIO Armed Conflict Dataset verzeichnet für Bolivien zwischen 1946 und 2010 drei interne bewaffnete Konflikte: den Sturz des reformistischen zivil-militärischen Regimes von Gualberto Villarroel 1946 (Krieg), die Revolution von 1952 (geringe Intensität) und die Auseinandersetzung der Putschregierung von René Barrientos und der Guerrilla um Che Guevara 1967 (geringe Intensität) (UCDO/PRIO 2011). Das Heidelberger Konfliktbarometer stuft die innerbolivianische Konfliktlage zwischen 2007 und 2010 als »Krise« ein (vgl. HIIK 2010) - für 2011 dürfte die Konfliktintensität abgesenkt werden.

17 Vgl. auch Gates et al. (2006); Hegre et al. (2001); Krain/Myers (1997); Spanger/Schesterinia (2012); Wolff (2009). 
stand unnötig bzw. unattraktiv macht; Letzteres impliziert die Annahme, dass die Existenz demokratischer Institutionen relative Deprivation bzw. grievances systematisch begrenzt. ${ }^{18}$

\subsection{Kausalmechanismus I: Demokratische Konfliktentschärfung}

Mit Blick auf den bolivianischen Transformationsprozess kann nicht die Rede davon sein, dass die Opposition - und konkret die regionalen Autonomiebewegungen ganz auf die Nutzung demokratischer Opportunitätsstrukturen setzte, um ihre Forderungen durchzusetzen. Wie gesehen, verfolgte sie regelmäßig alternative Strategien: außerinstitutionelle Proteste und Blockaden bis hin zur Besetzung und Plünderung staatlicher Institutionen auf der einen Seite, gegeninstitutionelle Strategien des Aufbaus regionaler Autonomieregierungen auf der anderen Seite. Gleichwohl spielten die demokratischen Institutionen ebenso eindeutig eine wichtige Rolle für den Verlauf der Auseinandersetzung. So nutzte die Opposition die eigene Blockademacht innerhalb des alten Kongresses sowie der Verfassunggebenden Versammlung systematisch, um eine politische Dominanz des MAS zu verhindern. Im Kongress setzte die Opposition, auf deren Zustimmung das Regierungslager angewiesen war, zunächst einen Modus für die Wahlen zur Asamblea Constituyente durch, der es dem MAS unmöglich machte, eine Zweidrittelmehrheit zu erreichen. Zugleich stritt sie - im Kongress und später in der Verfassunggebenden Versammlung kontinuierlich dafür, alle wichtigen Entscheidungen in der Versammlung an Zweidrittelmehrheiten zu binden.

Während diese Strategie im Rahmen der Constituyente letztlich scheiterte, war nach Abschluss der Versammlung - im Kongress schließlich erneut eine Zweidrittelmehrheit nötig, um das Verfassungsreferendum einzuberufen. ${ }^{19}$ Ein hinreichender Teil der parlamentarischen Opposition entschied sich - angesichts der extremen politischen Polarisierung und des drohenden Bürgerkriegs, aber auch der eindeutigen demokratischen Legitimation der Regierung - für den Versuch, eine friedliche und demokratische Lösung des Konflikts zu finden (Böhrt 2009: 60-61, 64). Die Verhandlungen im Kongress und das daraus resultierende Abkommen zwischen MAS und wichtigen Teilen der Opposition entsprechen weitgehend der Vorstellung demokratischer Konfliktbearbeitung: Sie bedeuteten den Wechsel des Konfliktaus-

18 Siehe hierzu grundlegend Wolff (2008: Kap. 2) sowie zusammenfassend Wolff (2009: 210-213).

19 Den Versuch der Regierung, das Referendum per Dekret am Kongress vorbei einzuberufen, wurde vom Nationalen Wahlgericht (Corte Nacional Electoral, CNE) untersagt. Dass sich die Regierung dem Einspruch der Judikative beugte, zeigt, dass deren Kontrollfunktion - trotz der oben angesprochenen Probleme - wenigstens noch partiell griff (vgl. Böhrt 2009: 63; Peñaranda 2009: 150-151; Romero 2009: 26). 
trags von gewaltträchtigen außerinstitutionellen zu demokratisch institutionalisierten Formen (Konfliktentschärfung) und ermöglichten dabei eine Kompromissbildung, die die dramatischen Positionsdifferenzen zwischen den Parteien deutlich reduzierte (Konfliktlinderung, siehe unten). ${ }^{20}$ Ein ähnlicher Prozess der erfolgreichen parlamentarischen Bearbeitung eines eskalierenden Konflikts ermöglichte im April 2009 die Verabschiedung eines vorläufigen Wahlgesetzes (und damit die Durchführung allgemein anerkannter Wahlen Ende des Jahres): Nach heftigen Auseinandersetzungen zwischen Regierungslager und Opposition, die in einem Hungerstreik des Präsidenten gipfelten, gelang im Kongress ein Kompromiss, der wesentliche Forderungen der Opposition enthielt. ${ }^{21}$

\subsection{Kausalmechanismus II: Demokratische Konfliktlinderung}

Der Verfassungskompromiss vom Oktober 2008 zeigt deutlich, dass demokratische Verfahren ihre konfliktentschärfende - d.h. die Formen des Konfliktaustrags transformierende - Wirkung nur dann entfalten können, wenn sie letztlich auch substanziell zur Bearbeitung der Konfliktursachen beitragen. Die Kongressverhandlungen auf der Basis der notwendigen Zweidrittelmehrheit boten den prozeduralen Rahmen, der eine friedliche Einigung ermöglichte. Dass das Abkommen in Folge auch tatsächlich befriedend wirkte, machte sich aber ebenso daran fest, dass ein politischer Kompromiss gelungen war, der von Regierungs- wie Oppositionslager als akzeptabel angesehen wurde - wenn ihn auch radikalere Gruppierungen auf beiden Seiten als Verrat kritisierten.

Die Überarbeitung der Verfassung kann insofern als zentrales Beispiel für eine effektive Linderung des Konflikts gelten, der die Bedrohungswahrnehmung - bzw. die Perzeption relativer Deprivation - auf Seiten der Opposition deutlich reduzierte. ${ }^{22}$ Dabei setzte die Opposition u.a. eine Klausel durch, die eine Wiederwahl von Präsident Morales untersagte. Die revidierte Verfassungsvorlage band eine Reihe von Entscheidungen an Zweidrittelmehrheiten im Parlament und schränkte plebiszitäre Entscheidungsmöglichkeiten (z.B. Schutz der Judikative vor Abberufungsreferenden) sowie die direkte Mitbestimmung der »organisierten Zivilgesellschaft« ein. Die Reichweite spezieller Wahlbezirke für indigene Völker und des indigenen

20 Nicht in das Bild institutionalisierter, demokratischer Konfliktbearbeitung passt allerdings der Druck, den geschätzte 100.000 MAS-Anhänger außerhalb des Parlaments ausübten (vgl. Jost 2009: 38; Peñaranda 2009: 185-186).

21 Zentrale Forderungen der Opposition waren eine biometrische Neuregistrierung der Wahlberechtigten sowie die Reduktion der Sondersitze für indigene Völker (vgl. La Razon 2009; El Pais 2009).

22 Vgl. Böhrt (2009: 65-105); Jost (2009: 39-41); Schilling-Vacaflor (2010: 214-217); Wolff (2012: 6-11). 
Gewohnheitsrechts wurden klar begrenzt. Dagegen wurden die Kompetenzen der Departments deutlich ausgeweitet. Schließlich wurde eine Bestimmung ergänzt, der zufolge die neu etablierte Obergrenze für Großgrundbesitz nicht auf bereits bestehenden Landbesitz anzuwenden ist. Auch bei den Verhandlungen um das vorläufige Wahlgesetz 2009 konnte die Opposition zentrale Forderungen durchsetzen (siehe oben).

Mit Blick auf die Umverteilung ökonomischer Ressourcen standen Landreform und die Einnahmen aus der Gasförderung im Zentrum der Auseinandersetzung. Für die Großgrundbesitzer und Agrarunternehmer in den Reihen der Autonomiebewegungen stellte die verfassungsrechtliche Ergänzung, dass die neue Obergrenze für Landbesitz nicht rückwirkend anzuwenden sei, ein zentrales Zugeständnis dar (Eaton 2011: 297). Konfliktlindernd wirkte zudem, dass die Regierung Morales in ihrer Agrarreformpolitik einen Schwerpunkt auf die Verteilung fiskalischer Böden legte (Urioste 2009: 124). Gleichwohl blieb dieses Politikfeld überaus konfliktträchtig, die Auseinandersetzungen verlagerten sich allerdings zunehmend von der nationalen auf die je lokale Ebene. In den Fällen, in denen sich die Regierung darum bemühte, Ländereien zu enteignen, die nicht die verlangte sozioökonomische Funktion erfüllten, kam es mitunter zu gewaltsamem Widerstand der Großgrundbesitzer (vgl. Ströbele-Gregor 2009: 148-149; Urioste 2009: 124). Neben dem Widerstand gegen den Verfassungsentwurf des MAS war es insbesondere die Verteilung der Gaseinnahmen, die die Proteste der Autonomiebewegungen im Jahr 2008 vorantrieb (vgl. Humphreys/Bebbington 2010: 147). Auslöser war die Entscheidung der Regierung Ende 2007, den Anteil der Department-Regierungen an einer Zusatzsteuer auf Kohlenwasserstoffe (Impuesto Directo a los Hidrocaburos, IDH) um 30\% zu reduzieren, um damit eine neue Universalrente zu finanzieren (Peñaranda 2009: 126; Romero 2009: 16). In diesem Fall konnten sich die Department-Regierungen nicht durchsetzen und mussten eine gewisse Reduktion ihrer Einnahmen hinnehmen. Aufgrund der generell deutlich erhöhten Erdgassteuern in einem Kontext tendenziell steigender Erdgaspreise griffen diese Einnahmeverluste allerdings auf relativ hohem Niveau und blieben vom Umfang her begrenzt (Fundación Jubileo 2009: 4-5, 10-11; vgl. Humphreys/Bebbington 2010).

\subsection{Voraussetzungen und Grenzen demokratischer Konfliktbearbeitung}

Es gehört zu einer demokratietheoretischen Standardwarnung, dass die Demokratie auf »moralischen Ressourcen « beruht, die sie nur in Grenzen selbst schaffen kann (Offe 2003: 327). In diesem Sinne zeigt das Konfliktverhalten von Regierung und Opposition Hinweise auf einen basalen Konsens über demokratische Grundregeln, 
der sich im Sinne von Larry Diamond (1990: 49) als Grundlage für das Funktionieren der oben geschilderten Mechanismen demokratischer Konfliktbearbeitung interpretieren ließe. Zwar griffen alle Konfliktparteien mitunter auf außerinstitutionelle - und teils gewaltgestützte - Formen des Konfliktaustrags zurück und mobilisierten insbesondere die eigenen Anhänger, um die Gegenseite unter Druck zu setzen (vgl. Eaton 2011; Laserna 2010; Peñaranda 2009). Allerdings setzte die Regierung die repressiven Instrumente des Staates nur sehr zurückhaltend ein (siehe unten); ein gewaltsames Vorgehen gegen die Autonomiebewegungen, das eine entsprechende Gewaltspirale hätte auslösen können, blieb entsprechend aus. Zugleich verfolgten die in der Opposition versammelten »alten Eliten « - im Unterschied zu früheren Jahrzehnten - nicht erkennbar eine Strategie des autoritären Putsches, sondern wählten mit der Forderung nach Autonomie einen im Kern demokratischen Weg, um die eigenen Interessen gegen die perzipierten »Angriffe« der Zentralregierung zu schützen (vgl. Eaton 2007; 2011). Inwieweit dies allerdings einen genuinen Basiskonsens spiegelt, ist unklar. Das Verhalten beider Seiten dürfte mindestens auch einer realistischen Einschätzung der jeweiligen Handlungsspielräume geschuldet sein, die sich insbesondere auf eine - in historischer Perspektive ungewöhnliche - politökonomische Konstellation zurückführen lässt: Das Militär, das als Teil der »alten Eliten « traditionell auf Seiten der Opposition zu verorten und für einen Putsch gegen Morales unabdingbar gewesen wäre, verhielt sich loyal gegenüber der Regierung und wendete sich klar gegen jegliche sezessionistischen Tendenzen; gleichzeitig verweigerte es sich aber ebenso einer repressiven Politik gegen die Tiefland-Opposition (siehe 4.5).

Gleichzeitig zeigt die reale Konflikteskalation, die Bolivien an den Rand eines Bürgerkriegs brachte, dass der wie auch immer genuine Basiskonsens über Grundregeln der Demokratie nur begrenzt wirksam war. Das Ausmaß politischer Polarisierung unter Bedingungen tendenziell überlappender cleavages $^{23}$ führte jedenfalls dazu, dass demokratische Verfahren in Bolivien - ganz im Sinne der Forschung, die die Risiken von Wahlen in gespaltenen Gesellschaften betont - nur zum Teil friedensfördernd, teilweise dagegen offen konfliktverschärfend wirkten. Schon ganz allgemein standen Wahlen und die Opportunitätsstrukturen, die sie boten, am Be-

23 Die Gegenüberstellung eines armen, indigen geprägten und auf den Zentralstaat und die Binnenwirtschaft ausgerichteten andinen Westens (Hochland), der von der Regierung Morales repräsentiert wird, und dem reichen, »weißen«, auf den Weltmarkt ausgerichteten Osten (Tiefland), den die oppositionellen Autonomiebewegungen vertreten, geht in vielerlei Hinsicht allzu grob über interne Fragmentierungen und gewichtige cross-cutting cleavages hinweg, erhielt aber in der Wahrnehmung der Akteure und im politischen Diskurs gleichwohl eine reale Bedeutung: als Konfrontation »der zwei Bolivien« (Dunkerley 2007; vgl. Eaton 2011: 300-302). 
ginn der Konfliktkonstellation: Politische Reformen in den 1990er Jahren, die Wahlbeteiligung und -erfolge des MAS ermöglichten, bildeten ein wichtiges Element im politischen Aufstieg der Partei bis hin zum Wahlsieg bei den Präsidentschaftswahlen Ende 2005. ${ }^{24}$ Wahlen wirkten damit einerseits in Richtung einer Vertiefung von Demokratie, insofern sie die Repräsentativität bzw. Inklusivität des politischen Systems deutlich steigerten, und dabei zugleich auch friedensfördernd, indem sie dazu beitrugen, den Widerstand der indigenen und sozialen Bewegungen in institutionelle Bahnen zu lenken. Andererseits bedeutete der Transformationsund Umverteilungsanspruch der bisher Marginalisierten allerdings, dass ihre Wahlerfolge die real-existierenden demokratischen Institutionen und konkret die alten Eliten offen herausforderten. Ab 2006 sah sich die Opposition auf nationaler Ebene zunehmend als strukturelle Minderheit, für die Wahlen und Referenden als Mittel der politischen Auseinandersetzung kaum mehr aussichtsreich schienen. ${ }^{25}$

In diesem Kontext trug die Verfassunggebende Versammlung - unabhängig von allen positiven Funktionen, die sie mit Blick auf die politische Inkorporation bisher marginalisierter Bevölkerungsteile besaß - mit Blick auf das Verhältnis zwischen Regierungslager und Opposition zu Polarisierung und Konflikteskalation bei. Folgerichtig endete sie Ende 2007 in offenen Konfrontationen. ${ }^{26}$ Auch ein weiterer Weg, den Konflikt auf demokratischem Wege zu entschärfen, scheiterte: Die Abberufungsreferenden im August 2008, in denen die wichtigsten politischen Kontra-

24 Hier sind insbesondere die Dezentralisierungs- und Volksbeteiligungsreformen zu nennen, die indigenen und sozialen Bewegungen über Wahlen auf lokaler Ebene einen formellen Zugang zum politischen System ermöglichten. Auf nationaler Ebene führten neu eingeführte Direktmandate dazu, dass geografisch konzentrierte Gemeinschaften (wie die Kokabauern um Evo Morales, die später den Kern des MAS bilden sollten) eigene Vertreter ins Parlament wählen konnten. Hinzu kamen Anstrengungen der Wählerregistrierung, die insbesondere die Wahlbeteiligung der (indigenen) Landbevölkerung deutlich steigerten (vgl. Van Cott 2005: 68-71, 87-95).

25 In Folge setzten die regionalen Autonomiebewegungen und ihre Vertretung in Kongress und Verfassunggebender Versammlung über weite Teile der Periode zwischen Antritt der MAS-Regierung im Januar 2006 und dem Verfassungsabkommen im Oktober 2008 auf institutionelle Blockade und Obstruktion einerseits, außer- und gegeninstitutionellen Widerstand andererseits (siehe hier insbesondere die Chronologie in Peñaranda 2009; vgl. auch Gamboa 2009; Schilling-Vacaflor 2010: Kap. 4).

26 Angesichts massiver Konflikte, die die Arbeit der in Sucre tagenden Verfassunggebenden Versammlung für Wochen unterbanden und zu zahlreichen Verletzten und drei Toten führten, wurde die Constituyente im Dezember 2007 zunächst in ein Militärgebäude nahe Sucre und schließlich nach Oruro verlegt, um die Verabschiedung der Vorlage zu ermöglichen. Weite Teile der Opposition blieben diesen letzten Sitzungen aus Protest fern (vgl. Gamboa 2009: 269; Peñaranda 2009: 127-134; Schilling-Vacaflor 2010: 145-147; Zegada 2010: 193). 
henten in ihren Ämtern bestätigt wurden, ${ }^{27}$ wirkten letztlich eher konfliktverschärfend. Dass Präsident Morales im doch eigentlich oppositionellen Tiefland teils überraschend gut abschnitt, ${ }^{28}$ signalisierte den Autonomiebewegungen, dass sie bei zukünftigen Wahlen (Verfassungsreferendum, Wahlen auf subnationaler Ebene) schlechte Karten haben dürften. Die These, dass dieses »Gefühl der Schwäche« auf Seiten der Opposition zu den »Ausbrüchen von Gewalt« im September 2008 beitrug (Peñaranda 2009: 152), ist durchaus plausibel.

\subsection{Kausalmechanismus III: Informelle Konfliktbearbeitung}

Die demokratischen Mechanismen der Konfliktbearbeitung erfassen zentrale Elemente des Aushandlungsprozesses zwischen Regierungslager und Opposition. Der Blick auf die formalen Institutionen weist allerdings insbesondere eine Blindstelle auf, die den Übergang zwischen außerinstitutioneller Konfrontation und institutioneller Verhandlung betrifft. Hier kommt die Bedeutung informeller Mechanismen der Konfliktbearbeitung ins Spiel. ${ }^{29}$

Dass demokratische Verhandlungen in Bolivien wie überall von diversen informellen Dialogen am Rande und außerhalb der formellen Institutionen begleitet werden, ist nicht weiter bemerkenswert. Am Beispiel des Verfassungskompromisses, ohne den der letztendlich friedliche Verlauf der politischen Transformation kaum denkbar ist, lässt sich aber zeigen, dass hier informelle Mechanismen der Konfliktbearbeitung unabdingbare Voraussetzungen des Verfassungskompromisses darstellten. Einerseits wurden die Verhandlungen im Kongress durch eine Serie direkter Gespräche überhaupt erst ermöglicht. Dies betrifft insbesondere den Dialog zwischen dem (relativ moderaten) Präfekten von Tarija, Mario Cossío, und der Regierung im September 2008, der seinerseits eine Verhandlung zwischen der Gruppe oppositioneller Präfekten und der Regierung ermöglichte, in der wichtige Weichenstellungen für den Verfassungskompromiss vorgenommen wurden (vgl. Gamboa 2009: 270-274; Peñaranda 2009: 165-183).

27 Bei den Referenden standen Präsident Morales sowie alle neun Präfekten des Landes zur Wahl. Lediglich zwei oppositionelle Präfekten in Departments, die nicht zum Tiefland gehören (Cochabamba, La Paz), wurden abgewählt. Morales erreichte landesweit eine Zustimmung von über 67\% (Romero 2009: 12-15).

28 In 27 der insgesamt 44 Provinzen der oppositionellen Departments stimmten Mehrheiten gegen eine Abberufung Morales'. In Pando setzte sich Morales sogar auf Departmentsebene durch und selbst in der Oppositionshochburg Santa Cruz erhielt Morales immerhin 40,7\% Zustimmung (Peñaranda 2009: 151).

29 Grundlegend zur Bedeutung informeller Institutionen in der Demokratie vgl. Helmke/Levitsky (2006); Lauth (2000). Für einen Vorschlag zur Integration informeller Mechanismen in eine erweiterte Perspektive auf den democratic civil peace vgl. Wolff (2009: 1004-1005; 2008: 85-102). 
Während sich in diesen Dialogprozessen immerhin anerkannte Repräsentanten der Konfliktparteien gegenübersaßen, erlangte die inhaltliche Vorbereitung des Verfassungskompromisses durch eine kleine Gruppe dialogbereiter Personen beider »Lager« lediglich ex post und de facto diese Bedeutung: Nach dem Ende der Verfassunggebenden Versammlung bemühte sich eine Gruppe um Carlos Romero, Abgeordneter des MAS in der Verfassunggebenden Versammlung und ab 2008 Minister, und Carlos Böhrt, Senator der Oppositionspartei PODEMOS, darum, Möglichkeiten eines konstitutionellen Kompromisses zwischen den unterschiedlichen Vorstellungen zu identifizieren. Sowohl die inhaltlichen Ergebnisse dieser Bemühungen (Alarcón et al. 2008; Romero et al. 2009) als auch das persönliche Vertrauen, das sie erzeugten, bildeten wichtige Grundlagen des parlamentarischen Verfassungskompromisses (vgl. Peñaranda 2009: 162).

Schließlich bedeuteten die Verhandlungen im Kongress zwar eine Rückkehr der Auseinandersetzung in formal-demokratische Bahnen - die (weitreichende) Überarbeitung des von der Verfassunggebenden Versammlung erarbeiteten Entwurfs durch das Parlament entbehrte allerdings jeglicher verfassungsrechtlicher Grundlage (Böhrt 2009: 65-66; Jost 2009: 38). Dass sich der Kongress - unter dem Einverständnis der zentralen Konfliktparteien und letztlich durch die Bevölkerung per Referendum sanktioniert - faktisch zum Congreso Constituyente erklärte und informell so handelte, als ob er diese Kompetenz besäße, war letztlich unabdingbare Bedingung des Verfassungskompromisses (vgl. Böhrt 2009: 66; Gamboa 2009: 270).

\subsection{Kausalmechanismus IV: Politökonomische Opportunitätsstruktur}

Die bisher analysierten Kausalmechanismen wirkten in Richtung friedlicher Konfliktbearbeitung, indem insbesondere die Motivation der Opposition zum Rückgriff auf Gewalt reduziert wurde: indem ihr formelle und informelle Möglichkeiten zur friedlichen Artikulation und partiellen Durchsetzung der eigenen Interessen und Werte geboten wurden. Bei allen prozeduralen und substanziellen Konzessionen, die die »alten Eliten« erreichen konnten, bleibt allerdings das Ausmaß, in dem sie politisch entmachtet wurden, in historischer und weltweit vergleichender Perspektive immer noch enorm. Um zu verstehen, warum der Widerstand der Opposition nach der Krise vom September 2008 gleichwohl begrenzt blieb, müssen deshalb neben den reduzierten Gründen auch die Möglichkeiten der Opposition zum Rückgriff auf Gewalt einbezogen werden. Diese Möglichkeiten wurden durch die spezifische politökonomische Opportunitätsstruktur, in der sich die »alten Eliten « unter der Regierung Morales wiederfanden, deutlich eingeschränkt. Dabei sind insbeson- 
dere drei zusammenhängende Merkmale zu nennen: die regionale Konzentration der Opposition, die empirische Legitimität der Regierung und der Mangel an (potenziellen) Alliierten der Autonomiebewegungen. ${ }^{30}$

Der MAS-Regierung stand von Beginn an eine auf nationaler Ebene äußerst fragmentierte Opposition gegenüber. Gleichzeitig waren die vom Regierungsprojekt bedrohten »alten Eliten « politisch und ökonomisch primär in den Tiefland-Departments des Landes verankert. Damit bot sich die regionale Autonomie als Abwehrstrategie der Opposition unmittelbar an (vgl. Eaton 2007; 2011). Diese regionale Konzentration verstärkte aber das Problem einer fehlenden Machtperspektive auf nationaler Ebene, insofern der quasi-nationalistische Autonomiediskurs breitere, landesweite Bündnisse erschwerte.

Das Gegenteil galt für die Regierung Morales, deren empirische Legitimität sowohl breit als auch tief war: breit, insofern sich die Regierung auf eine landesweit klar mehrheitliche Zustimmung (in Wahlen und Umfragen) stützen konnte, die auch im Tiefland noch signifikante Minderheiten (teilweise um die 40\%) umfasste; tief, insofern Morales mit der indigenen Bevölkerung und den sozialen und indigenen Bewegungen des Landes über eine Basis verfügte, die bereit war, »ihren« Präsidenten mit enormem Mobilisierungsaufwand zu verteidigen. Gleichzeitig verzichtete die Regierung, wie gesehen, weitgehend auf den Versuch, die Autonomiebewegungen per staatlicher Repression zu bekämpfen, was die Legitimität der Zentralregierung untergraben, eine auf »Gegengewalt« setzende Oppositionsstrategie gerechtfertigt und die Mobilisierungskraft der Autonomiebewegungen ausgeweitet hätte. Stattdessen waren es gesellschaftliche Kräfte, die im Namen der Regierung gegen die Autonomiebewegungen mobilisierten. Damit drehte sich die Konstellation um, wie sich zugespitzt im September 2008 zeigte: Es waren die oppositionellen Gruppierungen, die zur Gewalt gegen den Staat griffen und unbewaffnete Regierungsanhänger attackierten. Dies führte - wie sich spätestens nach dem »Massaker von Pando « zeigen sollte - zu deutlichen Legitimitätsverlusten der Opposition. Die Regierung Morales konnte sich dagegen als zurückhaltende, auf Frieden und nationale Einheit zielende Kraft darstellen, die erst in letzter Instanz - z.B. mit dem

30 Damit ist bereits gesagt, dass der Begriff der »politökonomischen Opportunitätsstruktur« hier keineswegs materialistisch verengt wird, sondern auch eine wichtige diskursive Dimension besitzt. Einerseits werden »materielle« Gegebenheiten - wie z.B. die ökonomische Basis der Opposition oder die politische/wirtschaftliche Interessenlage potenzieller Alliierter - erst in ihrer Wahrnehmung durch die Akteure als Opportunitäten und Beschränkungen wirksam. Andererseits hängen die politökonomischen Kräfteverhältnisse - etwa zwischen Regierung und Opposition - selbst in zentraler Weise davon ab, welche Forderungen, rhetorischen Figuren und Argumentationsmuster auf öffentliche Resonanz und Legitimität stoßen (vgl. hierzu grundlegend Koopmans/Olzak 2004). 
Ausnahmezustand in Pando - reagierte, um Gewaltexzesse der Autonomiebewegungen zu unterbinden (vgl. Peñaranda 2009: 161; Zegada 2010: 193).

Die regionale Konzentration der Autonomiebewegungen und die empirische Legitimität der Regierung führten schließlich dazu, dass der Opposition auf nationaler Ebene entscheidende Alliierte fehlten. Dies hat allgemein sicherlich mit einem starken nationalen (bzw. nationalistischen) Grundkonsens zu tun, der sich bis zur Revolution von 1952 zurückverfolgen lässt, an die die Agenda der Regierung Morales in vielerlei Hinsicht anschloss (vgl. Whitehead 2008: 262-268). ${ }^{31}$ An dieser Stelle ist insbesondere die bereits angesprochene Positionierung des Militärs von Bedeutung: Die spezifische politökonomische Konstellation, in der die linke und indigen geführte Regierung Morales ganz im Sinne der Identität der Streitkräfte für eine Agenda des ökonomischen und politischen Nationalismus stand, während die traditionelle Elite aus der Opposition eine tendenziell separatistische Strategie verfolgte, führte dazu, dass »die traditionellen Verbindungen zwischen Militär und den Eliten effektiv abgeschnitten wurden« (Kohl 2010: 111). Dem entsprachen auch konkrete materielle Interessen der Streitkräfte: Die Regierung Morales hatte signifikante Lohnerhöhungen und militärische Investitionen ermöglicht, die Forderung nach finanzieller Autonomie der Departments dagegen gefährdete tendenziell das Verteidigungsbudget, das aus zentralstaatlichen Mitteln gespeist wird (Kohl 2010: 111). ${ }^{32}$ Gleichzeitig weigerte sich die Führung der Streitkräfte aber offenbar, mit Repression gegen die Autonomiebewegungen vorzugehen (vgl. Lehoucq 2008: 122; US Embassy La Paz 2007; 2008). Dies hätte vorhersehbar zu einer Spaltung des Militärs mit potenziell hochkonfliktträchtigen Folgen geführt.

Die so umrissene politökonomische Opportunitätsstruktur bedeutete, dass für die Opposition letztlich weder Sezession noch Putsch eine realistische Perspektive darstellte. Das Ziel einer möglichen Strategie der gewaltsamen Konfrontation war damit völlig unklar, während das »Massaker von Pando« und seine politischen Folgen die absehbaren Kosten deutlich machten. In der Summe kann man mithin sagen, dass der friedliche Charakter des Umbruchs in Bolivien auch dadurch zu erklären ist, dass die »alten Eliten « jedenfalls zu einem Teil schlicht mit gewaltarmen Mitteln besiegt wurden. Nach dem Verfassungskompromiss vom Oktober 2008 und dem Referendum vom Januar 2009 war für die regionale Opposition klar, dass es realistisch nur mehr darum gehen konnte, bei der gesetzlichen Ausgestaltung der De-

31 Diesen Gedanken verdanke ich Hans-Jürgen Puhle.

32 Unter diesen Bedingungen blieb das Militär gegenüber der Regierung Morales loyal, »selbst nachdem er eine neue Generation von Generälen ernannt und erstmalig Indigene zur Offiziersschule der Streitkräfte zugelassen hatte« (Kohl 2010: 111). 
partment-Autonomien so viel wie möglich herauszuholen - was letztlich einen institutionalistischen Kurs verlangte.

\subsection{Kausalmechanismus V: Der internationale Kontext}

Obwohl das Konzept des democratic civil peace in vielerlei Hinsicht an das Paradigma des Demokratischen Friedens auf internationaler Ebene anschließt (vgl. Spanger/Schesterinia 2012), rekurrieren vorliegende Erklärungen zur innergesellschaftlichen Friedenswirkung der Demokratie in der Regel nicht systematisch auf internationale Faktoren. Im hier untersuchten Fall wirkte der internationale Kontext den friedlichen Verlauf des Umbruchs befördernd, indem externe Akteure die Versuche der formalen und informellen Konfliktbearbeitung unterstützten und zugleich die politökonomische Opportunitätsstruktur weiter zu Lasten einer auf Konfrontation setzenden Opposition verschoben.

Der politische Aufstieg, den Morales und der MAS seit Mitte der 1990er Jahren vollzogen, ist primär das Ergebnis innergesellschaftlicher Entwicklungen. Gleichwohl kann kaum bezweifelt werden, dass ihr bisheriger Erfolg an der Regierung, der sich nicht zuletzt in dem hier analysierten friedlichen Umbruch ausdrückt, von außergewöhnlichen internationalen - und vor allem regionalen - Rahmenbedingungen profitierte. Die seit 1990 im Rahmen der Organisation Amerikanischer Staaten (OAS) institutionalisierten Demokratienormen schlagen sich in einer sehr breiten regionalen Unterstützung gewählter Regierungen nieder (vgl. Hawkins/ Shaw 2007). Hinzu kam die spezifische Konjunktur des »Linksrucks« in Südamerika, der dazu führte, dass die politischen Sympathien für Morales in der Region hoch waren und die Regierung auf direkte Unterstützung insbesondere durch Venezuela, aber auch durch Argentinien und Brasilien zählen konnte (vgl. Schmalz 2009). Als die Regierung im September 2008 in ihre schwerste innenpolitische Krise geriet, reagierte die UNASUR mit einer eindeutigen Erklärung, in der sie die Regierung Morales unterstützte und die Aktionen der Autonomiebewegungen deutlich verurteilte. ${ }^{33}$ In Folge schloss sich die EU der UNASUR-Erklärung an (EU 2008), und auch die OAS erklärte ihre Unterstützung für die Regierung (Zegada 2010: 193). Diese Positionierung der internationalen Gemeinschaft, und besonders der UNASUR, bildete, so Carlos Romero (2009: 18), einen »Schlüsselfaktor, der zum

33 Die kurzfristig in Santiago de Chile versammelten Staatschefs der UNASUR erklärten der Regierung Morales ihre »vollste und entschiedenste Unterstützung«, kündigten an, dass sie jegliche Situation, die einen »Zivilputsch«, »einen Bruch der institutionellen Ordnung « oder eine Gefährdung »der territorialen Integrität« bedeuten würde, »energisch ablehnen und nicht anerkennen werden«, verurteilten »den Angriff auf Regierungseinrichtungen und auf die öffentliche Gewalt« und verurteilten aufs Schärfste »das Massaker« in Pando (zit. nach Peñaranda 2009: 160-161). 
Scheitern der Opposition beitrug« (vgl. Böhrt 2009: 59-60). Im November 2008 bestätigte eine Untersuchungskommission der UNASUR die Position der Regierung, dass es in Pando zu einem »Massaker « an unbewaffneten Regierungsanhängern gekommen sei, das einem»Verbrechen gegen die Menschlichkeit« gleichkäme (zit. nach Peñaranda 2009: 159). Zuvor hatten Beobachter der UNASUR, weiterer internationaler Organisationen sowie von Regierungen aus Lateinamerika und Europa die verschiedenen Runden des Dialogs zwischen Regierungslager und Opposition begleitet. Angesichts des extrem angespannten Klimas zwischen den Konfliktparteien wird die Bedeutung dieser externen Akteure als Vermittler und Beobachter als »vital« eingeschätzt (Peñaranda 2009: 176, 179; vgl. Böhrt 2009: 60, 63).

Die klare Positionierung der internationalen Gemeinschaft - und namentlich der UNASUR - zu Gunsten der Regierung Morales machte die Sezessionsdrohung der Autonomiebewegungen endgültig unglaubwürdig (Humphreys/Bebbington 2010: 153). ${ }^{34}$ Eine politische Anerkennung der regionalen Opposition innerhalb Südamerikas war damit ausgeschlossen. Und ökonomisch waren sowohl die Exportlandwirtschaft als auch vor allem der Gasexport unmittelbar von der Kooperation mit Argentinien und Brasilien abhängig. Schließlich fiel selbst die US-Regierung, der historisch wohl zuverlässigste Partner der bolivianischen Eliten, weitgehend aus. Während die Vereinigten Staaten vor dem Wahlsieg von Morales konsequent das regierende Establishment unterstützt und den MAS und insbesondere die Kokabauern um Morales offensiv bekämpft hatten, nahm die US-Regierung nach dem Wahlsieg von Morales eine deutlich ambivalentere Haltung ein. Jedenfalls entschied sie sich sowohl gegen eine offene Konfrontation mit der Regierung Morales als auch gegen eine offene Positionierung zu Gunsten der regionalen Autonomiebewegungen. Zwar diente eine Reihe von US-Aktivitäten der Unterstützung der oppositionellen Präfekturen, diese endete aber mit der Ausweisung des US-Botschafters im Kontext der innenpolitischen Krise im September 2008. ${ }^{35}$

\section{Schluss}

Bei aller Kritik, die üblicherweise am Zustand der bolivianischen Demokratie geübt wird (vgl. Bertelsmann Stiftung 2009; Gamarra 2008; Laserna 2010), erwies sich diese doch bemerkenswert fähig, einen dramatischen Machtübergang in weitgehend

34 Schon die eigenständig durchgeführten Autonomiereferenden in der media luna erreichten keinerlei internationale Zustimmung oder gar offizielle Anerkennung (Romero 2009: 11). Nach dem »Massaker von Pando« und der Positionierung der UNASUR war diese Option endgültig verschlossen.

35 Für eine ausführliche Analyse der US-Politik gegenüber Bolivien unter Morales siehe Wolff (2011). Vgl. auch Burron (2012). 
friedliche Bahnen zu lenken. Der überwiegend gewaltlose Verlauf des politischen Umbruchs, den Bolivien zwischen 2006 und 2009 erlebte, lässt sich zu guten Teilen auf das Funktionieren demokratischer Konfliktbearbeitung zurückführen: Demokratische Verfahren und Institutionen boten Möglichkeiten zum gewaltlosen Konfliktaustrag (Konfliktentschärfung) und sorgten zugleich dafür, dass die teils dramatischen Positionsdifferenzen zwischen Regierung und Opposition deutlich reduziert wurden (Konfliktlinderung). Zugleich bestätigte die Analyse, dass die Friedenswirkung der Demokratie unter Bedingungen struktureller Ungleichheit und konkret in einem Kontext politischer Polarisierung generell prekär ist und demokratische Verfahren mitunter auch konfliktverschärfend wirken können.

Einem analytisch-eklektizistischen Vorgehen folgend, wurden diese Ambivalenzen und Blindstellen eines eng verstandenen democratic civil peace in dreierlei Weise gefüllt. Erstens zeigten sich informelle Mechanismen der Konfliktbearbeitung als zentrales Bindeglied, um den Übergang von der außerinstitutionellen Konfrontation zur institutionellen Verhandlung zu füllen. Theoretisch verweist dies auf die Notwendigkeit, das Zusammenwirken formaler und informeller Konfliktbearbeitungsmodi systematisch in die Erklärung real-existierender Formationen des innergesellschaftlichen demokratischen Friedens einzubeziehen. Hier lässt sich an Arbeiten zur Bedeutung informeller Institutionen in der neueren Demokratieforschung anschließen (vgl. z.B. Helmke/Levitsky 2006; Lauth 2000).

Zweitens wurde mit Blick auf die politökonomische Opportunitätsstruktur herausgearbeitet, dass die »alten Eliten« trotz der verbleibenden Gründe zum notfalls gewaltsamen Widerstand gegen das Transformationsprojekt der Regierung nur in engen Grenzen über entsprechende Möglichkeiten verfügten: Während sich die Regierung Morales auf breite, landesweite Unterstützung, demokratische Legitimation und einen wirkmächtigen national-popularen Diskurs stützen konnte, blieb der Opposition eine regionalistische Abwehrstrategie, die ihre nationalen Handlungs- und Bündnisoptionen eng begrenzte. Daraus ergibt sich die theoretische Schlussfolgerung, dass neben einer erfolgreichen Konfliktbearbeitung auch die systematische Begrenzung der Konfliktfähigkeit soziopolitischer Akteure zur Aufrechterhaltung von Frieden beitragen kann. Üblicherweise sind es sozioökonomisch marginalisierte gesellschaftliche Schichten, die durch unterdurchschnittliche Kapazitäten zur politischen Artikulation und Mobilisierung von Widerstand charakterisiert sind dies gilt namentlich für die real-existierenden Demokratien Lateinamerikas (vgl. Wolff 2008; 2009). Ganz im Sinne der neueren sozialen Bewegungsforschung (vgl. z.B. McAdam et al. 2001) zeigt die vorliegende Fallstudie allerdings, dass Opportunitätsstrukturen nicht einfach in Gestalt eines bestimmten politischen Systems 
gegeben sind, sondern durch gesellschaftliches Handeln aktiv gestaltet und transformiert werden können.

Drittens erwies sich der internationale Kontext als Faktor, der die genannten innergesellschaftlichen Kausalmechanismen unterstützte: Vertreter der »internationalen Gemeinschaft « unterstützten einerseits die Versuche der formalen und informellen Konfliktbearbeitung und verschoben andererseits die politökonomische Opportunitätsstruktur weiter zu Lasten der Opposition. Diese innergesellschaftlich wie international geprägte politökonomische Opportunitätsstruktur ist dabei keineswegs rein materiell, sondern zu wichtigen Teil »diskursiv« (Koopmans/Olzak 2004): Die Verschiebung des Kräfteverhältnisses zu Gunsten der Regierung Morales - im Verhältnis zur regionalen Opposition - basierte nicht zuletzt auf der nationalen und externen Resonanz, auf die ihre politischen Forderungen, vor allem aber ihr Anspruch auf die legitime, demokratisch bestimmte Repräsentation des bolivianischen Volkes/Staates stießen. Mit Blick auf die kausale Bedeutung externer Akteure für die Reproduktion (oder Gefährdung) des innergesellschaftlichen Friedens weist der vorliegende Beitrag darauf hin, dass externe Akteure nicht erst durch die aktive Einmischung in das Konfliktgeschehen relevant werden, sondern auch dadurch, dass sie die materiellen Anreiz- und die ideellen Resonanzstrukturen beeinflussen, die das Denken und Handeln »lokaler« Akteure prägen.

In der Summe war es ein sehr spezifischer gesellschaftlicher und internationaler Kontext, der es der Regierung Morales erlaubte, gegen eine zentrale Erkenntnis der Demokratieforschung zu verstoßen: Die Stabilität der Demokratie unter Bedingungen struktureller sozialer Ungleichheit hängt davon ab, dass demokratische Verfahren »die dominanten Klassen gegen wahrgenommene Bedrohungen ihrer vitalen Interessen beschützen« (Rueschemeyer et al. 1992: 78; vgl. Wolff 2008: Kap. 2). Während formale und informelle Mechanismen der Konfliktbearbeitung das Ausmaß dieser Bedrohung nur partiell reduzierten, waren es die genannten innergesellschaftlichen und internationalen Kontextbedingungen, die die Optionen der bedrohten »alten Eliten « zur - notfalls gewaltsamen - Gegenwehr deutlich begrenzten. Der Blick auf die Kausalmechanismen, die das politische System formal bereitstellt, erwies sich mithin zwar als Einstieg und Rahmen analytisch hilfreich, als Erklärung war der Blick auf den democratic civil peace aber zu eng. Demokratie und ihre Fähigkeit zur friedlichen Bearbeitung soziopolitischer Konflikte sind nur zu verstehen, wenn demokratische Institutionen als eingebettet in und im Zusammenspiel mit informellen Politikmustern, politökonomischen Konfliktkonstellationen und internationalen Kontextbedingungen analysiert werden. Um dies herauszuarbeiten, bietet die Analyse signifikanter Nicht-Ereignisse - Episoden, in denen es bemerkenswerterweise nicht zu Gewalteskalation kam - einen fruchtbaren Zugang. Diese 
Forschungsstrategie fortsetzend ließen sich an die hier präsentierte Einzelfallstudie etwa komparative Fallstudien anschließen, in denen ähnlich gelagerte Episoden einsetzender und ausbleibender Gewalteskalation verglichen werden könnten.

Mit Blick auf Bolivien bleibt abschließend zu betonen, dass die vorliegende Analyse des friedlichen Umbruchs unter grundsätzlich demokratischen Vorzeichen keineswegs impliziert, dass die amtierende Regierung - normativ - als friedens- und/ oder demokratiepolitisch vorbildlich zu charakterisieren wäre. Während es in diesem Rahmen nur darum ging, den bemerkenswert gewaltarmen Verlauf des Großkonflikts zwischen Regierung und Opposition während der ersten Präsidentschaft von Evo Morales zu erklären, zeigen insbesondere die politischen Entwicklungen seit der Wiederwahl des Präsidenten, dass die Fähigkeit bzw. der Wille der Regierung zur konstruktiven Konfliktbearbeitung doch deutliche Grenzen aufweist. ${ }^{36}$ Nachdem sich die MAS-Regierung gegen die »alten Eliten « bis auf Weiteres durchgesetzt hat, eskalieren seit 2010 zunehmend Konflikte zwischen der Regierung und diversen Gruppierungen, die dem MAS eigentlich nahestehen bzw. -standen. Diese neue Konfliktwelle wird einerseits durch eine Vielzahl häufig widersprüchlicher Forderungen gesellschaftlicher Gruppen angetrieben, andererseits durch eine Regierung, die mitunter formale Verfahren der (präventiven) Konfliktbearbeitung missachtet und deren Dialogbereitschaft derzeit gering scheint. ${ }^{37}$ Dies wäre ein Thema für einen weiteren Artikel. Die vorliegende Analyse begründet lediglich die generelle Erwartung, dass der Regierung Morales im Prinzip hinreichende - formale wie informelle - Mechanismen der Konfliktbearbeitung zur Verfügung stehen sollten, um auch mit den gegenwärtigen Konflikten gewaltarm umgehen zu können.

\section{Literatur}

Alarcón Mondonio, Carlos/Böhrt Irahola, Carlos/Romero Bonifaz, Carlos Gustavo 2008: Hacia una Constitución democrática, viable y plural. Tres miradas, La Paz.

Anria, Santiago 2010: Bolivia's MAS: Between Party and Movement, in: Cameron, Maxwell A./Hershberg, Eric (Hrsg.): Latin America's Left Turns: Politics, Policies, and Trajectories of Change, Boulder, CO, 101-125.

36 Die Frage, wie die Demokratie unter Morales bzw. die Politik der MAS-Regierung aus demokratietheoretischer Sicht zu bewerten sind, war und ist nicht Thema dieses Artikels. Hierzu liegen erwartungsgemäß höchst unterschiedliche Analysen vor, die nicht zuletzt von den je partikularen Demokratievorstellungen der Autoren geprägt sind (vgl. z.B. Bertelsmann Stiftung 2009; 2012; Gamarra 2008; Laserna 2010; Vega 2010; Wolff 2012; Zegada et al. 2011).

37 Vgl. hierzu Mokrani (2011) und Zegada et al. (2011: 93-98) sowie detailliert die monatlichen Konfliktanalysen der Fundación UNIR Bolivia (z.B. UNIR Bolivia 2012). 
Ballentine, Karen/Sherman, Jake 2003: Introduction, in: dies. (Hrsg.): The Political

Economy of Armed Conflict: Beyond Greed and Grievance, Boulder, CO, 1-15. Bertelsmann Stiftung 2009: BTI 2010 - Bolivia Country Report, Gütersloh.

Bertelsmann Stiftung 2012: BTI 2012 - Bolivia Country Report, Gütersloh.

Böhrt Irahola, Carlos 2009: Cuarenta días que conmovieron a Bolivia y un pacto político forzado, in: Romero et al. 2009, 49-105.

Burron, Neil 2012: Unpacking U.S. Democracy Promotion in Bolivia: From Soft Tactics to Regime Change, in: Latin American Perspectives 39: 1, 115-132.

Bussmann, Margit/Hasenclever, Andreas/Schneider, Gerald 2009: Identität, Institutionen und Ökonomie: Ursachen und Scheinursachen innenpolitischer Gewalt, in: dies. (Hrsg.): Identität, Institutionen und Ökonomie. Ursachen innenpolitischer Gewalt, Wiesbaden, 9-35.

Cederman, Lars-Erik/Hug, Simon/Krebs, Lutz F. 2010: Democratization and Civil War: Empirical Evidence, in: Journal of Peace Research 47: 4, 377-394.

Collier, Paul 2009: Wars, Guns, and Votes. Democracy in Dangerous Places, New York, NY.

Crabtree, John/Whitehead, Laurence (Hrsg.) 2008: Unresolved Tensions: Bolivia Past and Present, Pittsburgh, PA.

Diamond, Larry 1990: Three Paradoxes of Democracy, in: Journal of Democracy 1: 3, 48-60.

Dunkerley, James 2007: Evo Morales, the »Two Bolivias« and the Third Bolivian Revolution, in: Journal of Latin American Studies 39: 1, 133-166.

Eaton, Kent 2007: Backlash in Bolivia: Regional Autonomy as a Reaction against Indigenous Mobilization, in: Politics \& Society 35: 1, 73-102.

Eaton, Kent 2011: Conservative Autonomy Movements: Territorial Dimensions of Ideological Conflict in Bolivia and Ecuador, in: Comparative Politics 43: 3, 291-310.

El País 2009: Morales acaba su ayuno tras el acuerdo en el Congreso, in: http:// www.ELPAIS.com; 15.4.2009.

Ernst, Tanja/Schmalz, Stefan (Hrsg.) 2009: Die Neugründung Boliviens? Die Regierung Evo Morales, Baden-Baden.

EU-European Union 2008: Declaration by the Presidency on behalf of the European Union on the Situation in Bolivia (19.9.2008), in: http://europa.eu/rapid/ searchAction.do; 20.1.2009.

Farthing, Linda/Kohl, Benjamin 2010: Social Control: Bolivia's New Approach to Coca Reduction, in: Latin American Perspectives 37: 4, 197-213.

Fearon, James D./Laitin, David 2003: Ethnicity, Insurgency, and Civil War, in: American Political Science Review 97: 1, 75-90. 
Freedom House 2011: Freedom in the World 2011: Bolivia, in: http://www.freedomhouse.org/report/freedom-world/2011/bolivia; 13.2.2012.

Fundación Jubileo 2009: Análisis del Presupuesto General de la Nación 2009, in: http://www.jubileobolivia.org.bo/recursos/files/pdfs/Analisis.pdf; 20.2.2012.

Gamarra, Eduardo A. 2008: Morales and Democracy, in: Domínguez, Jorge I./ Shifter, Michael (Hrsg.): Constructing Democratic Governance in Latin America, Third Edition, Baltimore, MD, 124-151.

Gamboa Rocabado, Franco 2009: Dilemas y conflictos sobre la Constitución en Bolivia. Historia Política de la Asamblea Constituyente, La Paz.

Gates, Scott/Hegre, Håvard/Jones, Mark P./Strand, Håvard 2006: Institutional Inconsistency and Political Instability: Polity Duration, 1800-2000, in: American Journal of Political Science 50: 4, 893-908.

Gromes, Thorsten 2007: Demokratisierung nach Bürgerkriegen. Das Beispiel Bosnien und Herzegowina, Frankfurt a.M.

Hawkins, Darren/Shaw, Carolyn M. 2007: The OAS and Legalizing Norms of Democracy, in: Legler, Thomas/Lean, Sharon F./Boniface, Dexter S. (Hrsg.): Promoting Democracy in the Americas, Baltimore, MD, 21-39.

Hegre, Håvard/Ellingsen, Tanja/Gates, Scott/Gleditsch, Nils Petter 2001: Toward a Democratic Civil Peace? Democracy, Political Change, and Civil War, 1816-1992, in: American Political Science Review 95: 1, 33-48.

Helmke, Gretchen/Levitsky, Steven (Hrsg.) 2006: Informal Institutions and Democracy. Lessons from Latin America, Baltimore, MD.

HIIK - Heidelberger Institut für Internationale Konfliktforschung 2010: Conflict Barometer 2010, in: http://www.hiik.de/en/konfliktbarometer/index.html; 24.6.2012.

Humphreys Bebbington, Denise/Bebbington, Anthony 2010: Anatomy of a Regional Conflict: Tarija and Resource Grievances in Morales's Bolivia, in: Latin American Perspectives 37: 4, 140-160.

Huntington, Samuel P. 1970: Political Order in Changing Societies, New Haven, CT.

Huntington, Samuel P. 1991: The Third Wave. Democratization in the Late 20th Century, Norman, OK.

IDEA Internacional (Hrsg.) 2010: Miradas: Nuevo Texto Constitucional, La Paz. Isidoro Losada, Ana María 2009: Alte Machthaber und aktuelle Opposition - Von den Zinnbaronen zu den Regionalfürsten im Tiefland, in: Ernst/Schmalz 2009, 95-108.

Jost, Stefan 2009: Kritische Anmerkungen zum bolivianischen Verfassungsprozess, in: Ernst/Schmalz 2009, 33-45. 
Jung, Dietrich 2005: Krieg als Geschäft? Nutzen und Kritik der ökonomischen Analyse, in: Jahn, Egbert/Fischer, Sabine/Sahm, Astrid (Hrsg.): Die Zukunft des Friedens, Band 2: Die Friedens- und Konfliktforschung aus der Perspektive der jüngeren Generation, Wiesbaden, 269-290.

Käss, Susanne/Velásquez Castellanos, Iván (Hrsg.) 2009: Reflexión Crítica a la nueva Constitución Política del Estado, La Paz.

Kaup, Brent Z. 2010: A Neoliberal Nationalization? The Constraints on NaturalGas-Led Development in Bolivia, in: Latin American Perspectives 37: 3, 123-138.

Kohl, Benjamin 2010: Bolivia under Morales: A Work in Progress, in: Latin American Perspectives 37: 3, 107-122.

Kohl, Benjamin/Bresnahan, Rosalind (Hrsg.) 2010: Bolivia Under Morales, Latin American Perspectives 37: 3-4.

Koopmans, Ruud/Olzak, Susan 2004: Discursive Opportunities and the Evolution of Right-Wing Violence in Germany, in: American Journal of Sociology 10: 1, 198-230.

Krain, Matthew/Myers, Marissa Edson 1997: Democracy and Civil War: A Note on the Democratic Peace Proposition, in: International Interactions 23: 1, 109-118.

Laserna, Roberto 2010: Mire, la democracia boliviana, en los hechos..., in: Latin American Research Review 45: Special Issue, 27-58.

La Razón 2009: Ley define voto en el exterior y 7 escaños para indígenas, in: http:// www.La-Razon.com; 14.4.2009.

Lauth, Hans-Joachim 2000: Informal Institutions and Democracy, in: Democratization 7: 4, 21-50.

Lehoucq, Fabrice 2008: Bolivia's Constitutional Breakdown, in: Journal of Democracy 19: 4, 111-124.

Lessmann, Robert 2010: Das neue Bolivien. Evo Morales und seine demokratische Revolution, Zürich.

Linz, Juan J. 1978: The Breakdown of Democratic Regimes: Crisis, Breakdown, and Reequilibration, Baltimore, MD.

Mansfield, Edward D./Snyder, Jack 2008: Democratization and Civil War (Saltzman Working Paper 5), in: http://www.siwps.com/programs/SWP.html; 31.8.2009.

Marshall, Monty G./Jaggers, Keith 2011: Polity IV Country Report 2010: Bolivia, in: http://www.systemicpeace.org/polity/Bolivia2010.pdf; 13.2.2012.

Matthies, Volker 1994: Friedensursachenforschung. Ein vernachlässigtes Feld, in: Wissenschaft \& Frieden 12: 2, 45-49. 
Mayorga, René Antonio 1997: Bolivia's Silent Revolution, in: Journal of Democracy $8: 1,142-156$.

McAdam, Doug/Tarrow, Sidney/Tilly, Charles 2001: Dynamics of Contention, Cambridge.

Mendonça Cunha, Clayton/Santaella Gonçalves, Rodrigo 2010: The National Development Plan as a Political Economic Strategy in Evo Morales's Bolivia: Accomplishments and Limitations, in: Latin American Perspectives 37: 4, 177-196.

Mokrani, Dunia 2011: Konfliktszenarien in der zweiten Amtszeit von Präsident Evo Morales (Rosa Luxemburg Stiftung Papers, Oktober 2011), in: http://www.rosalux.de/publication/37894/konfliktszenarien-in-der-zweiten-amtszeit-von-praesident-evo-morales.html; 19.10.2011.

Newman, Edward/Paris, Roland/Richmond, Oliver P. (Hrsg.) 2009: New Perspectives on Liberal Peacebuilding, Tokio.

Offe, Claus 2003: Herausforderungen der Demokratie. Zur Integrations- und Leistungsfähigkeit politischer Institutionen, Frankfurt a.M.

Peñaranda Undurraga, Raúl 2009: Crónica del proceso constituyente, in: Romero et al. 2009, 107-201.

PNUD - Programa de las Naciones Unidas para el Desarrollo 2010 a: Los cambios detrás del cambio. Informe Nacional sobre Desarrollo Humano en Bolivia, La Paz.

PNUD - Programa de las Naciones Unidas para el Desarrollo 2010 b: Mutaciones del campo político en Bolivia, La Paz.

Puhle, Hans-Jürgen 2001: Herausragende Transformations- und Entwicklungsleistungen in Bolivien, in: Weidenfeld, Werner (Hrsg.): Den Wandel gestalten Strategien der Transformation, Band 1, Gütersloh, 169-187.

Romero Bonifaz, Carlos 2009: Bolivia: de la confrontación al pacto político, in: Romero et al. 2009, 9-48.

Romero Bonifaz, Carlos/Böhrt Irahola, Carlos/Peñaranda Undurraga, Raúl (Hrsg.) 2009: Del conflicto al diálogo. Memorias del acuerdo constitucional, La Paz.

Rueschemeyer, Dietrich/Huber, Evelyne/Stephens, John D. 1992: Capitalist Development and Democracy, Cambridge.

Sambanis, Nicholas 2005: Conclusion: Using Case Studies to Refine and Expand the Theory of Civil War, in: Collier, Paul/Sambanis, Nicholas (Hrsg.): Understanding Civil War. Evidence and Analysis, Volume 1: Africa, Washington, DC, 303-334.

Schilling-Vacaflor, Almut 2010: Recht als umkämpftes Terrain. Die neue Verfassung und indigene Völker in Bolivien, Baden-Baden. 
Schmalz, Stefan 2009: Boliviens außenpolitische Umorientierung: Auf dem Weg zu einem südamerikanischen Linksblock?, in: Ernst/Schmalz 2009, 219-234.

Sil, Rudra/Katzenstein, Peter J. 2010: Beyond Paradigms. Analytic Eclecticism in the Study of World Politics, Houndmills.

Snyder, Jack 2000: From Voting to Violence. Democratization and Nationalist Conflict, New York, NY.

Spanger, Hans-Joachim/Schesterinia, Anastasia 2012: Demokratischer Frieden nach außen und innen? Der Forschungsstand zum Civil Democratic Peace, in: Spanger, Hans-Joachim (Hrsg.): Der demokratische Unfrieden. Über das spannungsreiche Verhältnis zwischen Demokratie und innerer Gewalt, Baden-Baden, 9-34.

Stefanoni, Pablo 2009: Indianismo y nacionalismo revolucionario: un análisis del gobierno de Evo Morales, in: Ospina, Pablo/Kaltmeier, Olaf/Büschges, Christian (Hrsg.): Los Andes en movimiento. Identidad y poder en el nuevo paisaje político, Quito, 95-106.

Ströbele-Gregor, Juliana 2009: Kampf um Land, in: Ernst/Schmalz 2009, 141-153. UCDP/PRIO 2011: UCDP/PRIO Armed Conflict Dataset v. 4-2011, 1946-2010, in: http://www.pcr.uu.se/research/ucdp/datasets/ucdp_prio_armed_conflict_dataset; 14.2.2012.

UNIR Bolivia 2012: Informe de seguimiento y análisis de la conflictividad en Bolivia. Diciembre de 2011, in: http://www.unirbolivia.org/nueva3/images/stories/ analisisdeconflictos/Info_Conflic_DICIEMBRE_2011_def.pdf; 27.6.2012.

Urioste, Miguel 2009: La »revolución agraria« de Evo Morales: desafíos de un proceso complejo, in: Nueva Sociedad 223/2009, 113-127.

US Embassy La Paz 2007: Cable 07LAPAZ3262, Military Standdown More Likely Than Heavy Hand, in: http://wikileaks.vicepresidencia.gob.bo/index.php?codigo=07LAPAZ3262; 8.12.2010.

US Embassy La Paz 2008: Cable 08LAPAZ483, Would Bolivian Military Go Along With a Crackdown?, in: http://www.wikileaks.ch/cable/2008/03/08LAPAZ483.html; 1.11.2011.

Van Cott, Donna Lee 2005: From Movements to Parties in Latin America. The Evolution of Ethnic Politics, Cambridge.

Vega Camacho, Oscar 2010: Transformaciones, descolonización y comunidades, in: Lang, Miriam/Santillana, Alejandra (Hrsg.): Democracia, participación y socialismo. Bolivia - Ecuador - Venezuela, Quito, 87-93.

Weisbrot, Mark/Ray, Rebecca/Johnston, Jake 2009: Bolivia: The Economy During the Morales Administration, in: http://www.cepr.net/documents/publications/ bolivia-2009-12.pdf; 15.4.2010. 
Whitehead, Laurence 2008: Conclusion: Bolivia’s Latest »Refoundation«, in: Crabtree/Whitehead 2008, 255-269.

Wimmer, Andreas/Schetter, Conrad 2003: Ethnic Violence, in: Heitmeyer, Wilhelm/Hagan, John (Hrsg.): International Handbook of Violence Research, Dordrecht, 247-260.

Wolff, Jonas 2008: Turbulente Stabilität. Die Demokratie in Südamerika diesseits ferner Ideale, Baden-Baden.

Wolff, Jonas 2009: De-Idealizing the Democratic Civil Peace: On the Political Economy of Democratic Stabilisation and Pacification in Argentina and Ecuador, in: Democratization 16: 5, 998-1026.

Wolff, Jonas 2011: Challenges to Democracy Promotion: The Case of Bolivia (Carnegie Paper, March 2011), in: http://carnegieendowment.org/files/democracy_bolivia.pdf; 10.2.2012.

Wolff, Jonas 2012: Towards Postliberal Democracy in Latin America? A Conceptual Framework Applied to Bolivia, in: Journal of Latin American Studies, i.E. Zegada, María Teresa 2010: El rol de la oposición política en Bolivia (2006-2009), in: PNUD 2010 b, 151-239.

Zegada, María Tereza/Arce, Claudia/Canedo, Gabriela/Quispe, Alber 2011: La democracia desde los márgenes. Transformaciones en el campo político boliviano, La Paz.

\section{Der Autor}

Dr. Jonas Wolff ist wissenschaftlicher Mitarbeiter der Hessischen Stiftung Friedens- und Konfliktforschung (HSFK) in Frankfurt a.M.

E-Mail: wolff@hsfk.de 